7?

columns any simple term that could describe 'strange' speech.

Currently there are terms that only partly describe what we wanted to be included in a single general term. For example, schizophrenic speech is composed of unusual oral creations which cannot be considered as a language as the latter is nothing if it is not creative. ${ }^{1}$ The term 'schizophasia' designates, specifically, at least two forms of unconventional surface speech behaviours - 'glossomanic behaviour' and 'glossolalic behaviour' that can be observed in certain patients who experience a psychotic episode. ${ }^{2}$ Both can be spectacular. The essential characteristics of 'glossomanic schizophasia' is the production of utterances the linguistic components of which - be they phonemes, words or more complex units - are selected and combined on the basis of superficial or semantic kinships rather than an immediately shareable topic. The main characteristic of 'glossolalic schizophasia' is an entirely or nearly entirely neologistic discourse.

However, the Greek term xenophonia describes what we are looking for. The exact definition for xenophonia is any strange/odd/paradoxical voice or speech $^{3,4}$ and xenophonic is one who speaks or sounds strange. Following a thorough research of all available databases, including EMBASE, MEDLINE and PsycINFO, without any language restriction, the term xenophonia has been referred to in only one paper, a non-psychiatric study. It is being used there to describe a vocal abnormality during and after the sound variation stage; the main symptoms are high tone, low voice, short breath and unstable sound control, which are usually a functional variation, a habitual vocal defect. $^{5}$

As the term xenophonia has never been mentioned in our fields of interest we would like to propose it as a new psychiatric term which describes the phenomena of generally 'strange speech'

1 Sims A. Speech and Language Disorders in Psychiatry. Gaskell, 1995.

2 Lecours AR, Navet M, Ross A. Langage et pensée du schizophase. Confrontations Psychiatriques 1981; 19: 109-44

3 Liddell HG, Scott R, Jones HS, McKenzie R. A Greek-English Lexicon. Clarendon Press, 1940

4 Dimitrakou D. Grand Dictionary of the Greek Language, Vol. 10, 1964

5 Zhiqing W, WenjunY, Yiting C. Tratment of xenophonia in male youths by extralaryngeal massage and language training. J Tradit Chin Med 1993; 13: 221-2.

*Panagiotis Zis PhD student in neurology, University of Athens, CT2 in Psychiatry, London Deanery, University of Athens, 22-26 Daskalak Street,11526 Athens, Greece, email: takiszis@ gmail.com, Bryan C. Timmins Consultant
Neuropsychiatrist, Northamptonshire Healthcare NHS Trust

doi: 10.1192/pb.33.7.275b

\section{ECT: there is more than just unilateral or bilateral selection!}

The assumption that all doctors are well informed about the latest arguments regarding the pros and cons of unilateral or bilateral electroconvulsive therapy (ECT) may not be right. We would like to take this opportunity to update readers of current developments that may potentially revolutionise or even significantly modify our thinking about this controversial treatment.

As the author says, the UK ECT review group in 2003 had an important short coming of inclusion of all stimulus intensities, leading to a dubious conclusion in favour of the advantages of bilateral ECT. Although we do believe that the uncertainty in evidence exists, the emerging evidence base, particularly in the USA and Australia, may tilt the balance of opinion and attitudes, more in favour of right unilateral (RUL) ECT with the ultra-brief type of pulse width.

Sackeim et $a l^{2}$ and Loo et al ${ }^{3}$ have in 2008 published research indicating that ultra-brief pulse width right unilateral ECT is likely as effective as the conventional one (brief pulse RUL), in addition to being significantly better in terms of cognitive disability. This is an exciting new development as we believe cognitive disability has consistently been underplayed in studies on ECT over the years. Robertson \& Pryor $^{4}$ as well as Mangaoang \& Lucey $^{5}$ cite extensive relevant body of research suggesting a lot more cognitive damage and disability, undetected by conventional testing. Additionally, if the patients were to be made aware of a potential modality of treatment with significantly less cognitive disability, they may actually make a more completely informed decision.

Although it is not difficult to adapt current practice to using ultra-brief pulse width RUL ECT by slight modification of the 'programmes' settings available on current machines in the UK, this detail is clearly beyond the scope of this letter.

In conclusion, we posit that the need for faster recovery by using bilateral ECT may be more than balanced by the need to deliver the treatment that is less disabling (in terms of cognitive disability) and possibly equally effective.

1 The UK ECT Review Group. Electroconvulsive therapy: systematic review and meta-analysis of efficacy and safetly in depressive disorders. Lancet 2003: 361:799-808.

2 Sackeim HA, Prudic P, Nobler MS, Fitzsimmons L, Lisanby SH, Payne N, et al. Effects of pulse width and electrode placement on the efficacy and cognitive effects of electroconvulsive therapy. Brain Stimulat 2008; 1: 71-83.

3 Loo CK, Sainsbury K, Sheehan P, Lyndon B. A comparison of RUL ultrabrief pulse (0.3 ms) ECTand standard RUL ECT. Int J Neuropsychopharmacol 2008; 11: 883-90.

4 Robertson H, Pryor R. Memory and cognitive effects of ECT: informing and assessing patients. Advan Psychiatr Treat 2006; 12: 228-37.

5 Mangaoang MA, LuceyJV. Cognitive rehabilitation: assessment and treatment of persistent memory impairments following ECT. Advan PsychiatrTreat 2007; 13: 90-100

*Mukesh Kripalani Specialist Registrar in Adult Psychiatry, Northern Deanery, Tees, Esk and Wear Valleys NHS Foundation Trust, email: drmukesh@ doctors.org.uk, Vinod Chaugule Consultant Psychiatrist and ECT Lead for Tees, Esk and Wear Valleys NHS Foundation Trust

doi: 10.1192/pb.33.7.276

\section{A survey on takeaways in a secure unit}

Physical health monitoring of long-term detained psychiatric patients in secure care has attracted much attention in the past few years. ${ }^{1}$ The rate of coronary heart disease in patients with schizophrenia is almost three times higher than in the general population and is thought to be a greater contributor to mortality in this group of patients than suicide. ${ }^{2}$ Patients on antipsychotic medication seem to have a worse metabolic profile. ${ }^{3}$ Metabolic syndrome has been described as a risk factor associated with the development of coronary heart disease and includes central obesity, impaired glucose tolerance, hypertriglyceridaemia, hypercholesterolaemia and hypertension.

For long-stay patients in secure hospitals a combination of antipsychotic medication, poor diet, sedentary lifestyle, lack of exercise and leave, smoking and illness effects are all likely to contribute to weight gain and metabolic syndrome.

As part of a wider consultation exercise promoting healthy lifestyles, concern has been raised about the number of takeaways ordered by detained patients within a National Health Service (NHS) medium secure unit and how this may contribute to metabolic syndrome. A survey monitored the number of takeaways delivered to the unit over a 21-day period.

In total, 326 individual takeaways at the overall cost of $£ 2736$ were consumed at an average of $£ 8.40$ per order (range f3-23). The figures included 'group bookings' from two wards within the learning disability directorate that have two designated takeaway nights per week.

It was estimated that around threequarters of patients ordered a takeaway during the study period: 29 patients consumed at least one takeaway a week and 16 patients consumed at least 